KYUNGPOOK Math. J. 54(2014), 531-543

http://dx.doi.org/10.5666/KMJ.2014.54.4.531

\title{
Some Properties Subclasses of Analytic Functions
}

BASEM AREF FRASIN

Faculty of Science, Department of Mathematics, Al al-Bayt University, P. O. Box: 130095 Mafraq, Jordan

e-mail : bafrasin@yahoo.com

Abstract. The object of the present paper is to discuss some interesting properties of analytic functions $f(z)$ associated with the subclasses $\mathcal{D}\left(\beta_{1}, \beta_{2}, \beta_{3} ; \lambda\right), \mathcal{G}(\theta, \alpha)$ and $\mathcal{Q}(\theta, \alpha)$. Also, radius problems of $\frac{1}{\delta} f(\delta z)$ for $f(z)$ in the class $\mathcal{D}\left(\beta_{1}, \beta_{2}, \beta_{3} ; \lambda\right), \mathcal{G}(\theta, \alpha)$ and $\mathcal{Q}(\theta, \alpha)$ are considered.

\section{Introduction and Definitions}

Let $\mathcal{A}$ denote the class of the normalized functions of the form

$$
f(z)=z+\sum_{n=2}^{\infty} a_{n} z^{n}
$$

which are analytic in the open unit disk $\mathcal{U}=\{z \in \mathbb{C}:|z|<1\}$. For a function $f(z)$ in the class $\mathcal{A}$, Sãlãgean [1] defined the differential operator $D^{k}$, by

$$
\begin{aligned}
D^{0} f(z) & =f(z), \\
D^{1} f(z) & =D f(z)=z f^{\prime}(z), \\
D^{2} f(z) & =D\left(D^{1} f(z)=z\left(z f^{\prime}(z)\right)^{\prime},\right.
\end{aligned}
$$

and

$$
D^{k} f(z)=D\left(D^{k-1} f(z)\right), \quad(k \in \mathbb{N}) .
$$

Thus

$$
D^{k} f(z)=z+\sum_{n=2}^{\infty} n^{k} a_{n} z^{n}, \quad(k \in \mathbb{N} \cup\{0\}) .
$$

Received October 13, 2011; accepted March 27, 2013.

2010 Mathematics Subject Classification: 30C45.

Key words and phrases: Analytic, univalent functions, Cauchy-Schwarz inequality, radius problems. 
Let $\mathcal{G}(\alpha)$ denote the subclass of $\mathcal{A}$ consisting of functions $f(z)$ which satisfy

$$
\operatorname{Re}\left\{\frac{\mathrm{D}^{3} \mathrm{f}(\mathrm{z})}{\mathrm{z}}\right\}>\alpha
$$

for some $\alpha(0 \leq \alpha<1)$ and for all $z \in \mathcal{U}$. Also, Let $\mathcal{Q}(\alpha)$ denote the subclass of $\mathcal{A}$ consisting of functions $f(z)$ which satisfy

$$
\operatorname{Re}\left\{\frac{D^{2} f(z)}{D^{1} f(z)}\right\}>\alpha
$$

for some $\alpha(0 \leq \alpha<1)$ and for all $z \in \mathcal{U}$.

For analytic functions $f(z)$, Uyanik and Owa [2], obtained some interesting properties for analytic functions in the subclass $\mathcal{A}\left(\beta_{1}, \beta_{2}, \beta_{3} ; \lambda\right)$ defined by

$$
\begin{gathered}
\left|\beta_{1} z\left(\frac{f(z)}{z}\right)^{\prime}+\beta_{2} z^{2}\left(\frac{f(z)}{z}\right)^{\prime \prime}+\beta_{3} z^{3}\left(\frac{f(z)}{z}\right)^{\prime \prime \prime}\right| \leq \lambda \\
\left(\beta_{1}, \beta_{2}, \beta_{3} \in \mathbb{C} ; \lambda>0 ; z \in \mathcal{U}\right),
\end{gathered}
$$

associated with close-to-convex functions and starlike functions of order $\alpha$.

In this paper, we define the following subclass of analytic functions.

Definition 1.1. A function $f(z)$ belonging to $\mathcal{A}$ is said to be in the class $\mathcal{D}\left(\beta_{1}, \beta_{2}, \beta_{3} ; \lambda\right)$ if it satisfies

(4) $\left|\beta_{1} z\left(\frac{D^{2} f(z)}{z}\right)^{\prime}+\beta_{2} z^{2}\left(\frac{D^{2} f(z)}{z}\right)^{\prime \prime}+\beta_{3} z^{3}\left(\frac{D^{2} f(z)}{z}\right)^{\prime \prime \prime}\right| \leq \lambda \quad(z \in \mathfrak{U})$,

for some complex numbers $\beta_{1}, \beta_{2}, \beta_{3}$, and for some real $\lambda>0$.

Example 1.2. Let us consider the function $f_{\gamma}(z), \gamma \in \mathbb{R}$, given by

$$
f_{\gamma}(z)=z(1+z)^{\gamma}
$$

then, we have

$$
D^{2} f_{\gamma}(z)=z+\sum_{n=2}^{\infty} n^{2}\left(\begin{array}{c}
\gamma \\
n-1
\end{array}\right) z^{n}
$$

where

$$
\left(\begin{array}{c}
\gamma \\
n-1
\end{array}\right)=\frac{\gamma(\gamma-1)(\gamma-2) \ldots(\gamma-n+2)}{(n-1) !}
$$


From (5), it follows that

$$
\begin{aligned}
& \left|\beta_{1} z\left(\frac{D^{2} f_{\gamma}(z)}{z}\right)^{\prime}+\beta_{2} z^{2}\left(\frac{D^{2} f_{\gamma}(z)}{z}\right)^{\prime \prime}+\beta_{3} z^{3}\left(\frac{D^{2} f_{\gamma}(z)}{z}\right)^{\prime \prime \prime}\right| \\
= & \left|\sum_{n=2}^{\infty} n^{2}(n-1)\left(\begin{array}{c}
\gamma \\
n-1
\end{array}\right)\left(\beta_{1}+(n-2) \beta_{2}+(n-2)(n-3) \beta_{3}\right) z^{n-1}\right| .
\end{aligned}
$$

Therefore, if $\gamma=1$, then

$$
\left|\beta_{1} z\left(\frac{D^{2} f_{1}(z)}{z}\right)^{\prime}+\beta_{2} z^{2}\left(\frac{D^{2} f_{1}(z)}{z}\right)^{\prime \prime}+\beta_{3} z^{3}\left(\frac{D^{2} f_{1}(z)}{z}\right)^{\prime \prime \prime}\right|=\left|4 \beta_{1} z\right| \leq 4\left|\beta_{1}\right| .
$$

This implies that $f_{1}(z) \in \mathcal{D}\left(\beta_{1}, \beta_{2}, \beta_{3} ; \lambda\right)$ for $\lambda \geq 4\left|\beta_{1}\right|$. If $\gamma=2$, then

$$
\begin{aligned}
& \left|\beta_{1} z\left(\frac{D^{2} f_{2}(z)}{z}\right)^{\prime}+\beta_{2} z^{2}\left(\frac{D^{2} f_{2}(z)}{z}\right)^{\prime \prime}+\beta_{3} z^{3}\left(\frac{D^{2} f_{2}(z)}{z}\right)^{\prime \prime \prime}\right| \\
= & \left|8 \beta_{1} z+18\left(\beta_{1}+\beta_{2}\right) z^{2}\right| \leq 26\left|\beta_{1}\right|+18\left|\beta_{2}\right| .
\end{aligned}
$$

Therefore, $f_{2}(z) \in \mathcal{D}\left(\beta_{1}, \beta_{2}, \beta_{3} ; \lambda\right)$ for $\lambda \geq 26\left|\beta_{1}\right|+18\left|\beta_{2}\right|$. Further, if $\gamma=3$, then we have

$$
\begin{aligned}
& \left|\beta_{1} z\left(\frac{D^{2} f_{3}(z)}{z}\right)^{\prime}+\beta_{2} z^{2}\left(\frac{D^{2} f_{3}(z)}{z}\right)^{\prime \prime}+\beta_{3} z^{3}\left(\frac{D^{2} f_{3}(z)}{z}\right)^{\prime \prime \prime}\right| \\
= & \left|12 \beta_{1} z+54\left(\beta_{1}+\beta_{2}\right) z^{2}+48\left(\beta_{1}+2 \beta_{2}+2 \beta_{3}\right) z^{3}\right| \\
\leq & 114\left|\beta_{1}\right|+150\left|\beta_{2}\right|+96\left|\beta_{3}\right| .
\end{aligned}
$$

Thus, $f_{3}(z) \in \mathcal{D}\left(\beta_{1}, \beta_{2}, \beta_{3} ; \lambda\right)$ for $\lambda \geq 114\left|\beta_{1}\right|+150\left|\beta_{2}\right|+96\left|\beta_{3}\right|$.

Now, let $\mathcal{A}_{\theta}$ denote the subclass of $\mathcal{A}$ consisting of functions $f(z)$ with

$$
a_{n}=\left|a_{n}\right| e^{i((n-1) \theta+\pi)} \quad(n=2,3, \ldots) .
$$

Also, we introduce the subclasses $\mathcal{G}(\theta, \alpha)$ and $\mathcal{Q}(\theta, \alpha)$ of $\mathcal{A}_{\theta}$ as follows:

$$
\mathcal{G}(\theta, \alpha)=\mathcal{A}_{\theta} \cap \mathcal{G}(\alpha) \text { and } \mathcal{Q}(\theta, \alpha)=\mathcal{A}_{\theta} \cap \mathcal{Q}(\alpha) .
$$


2. Properties of the Class $\mathcal{D}\left(\beta_{1}, \beta_{2}, \beta_{3} ; \lambda\right)$

We first prove

Theorem 2.1. If $f(z) \in \mathcal{A}$ satisfies

$$
\sum_{n=2}^{\infty} n^{2}(n-1)\left(\left|\beta_{1}\right|+(n-2)\left|\beta_{2}\right|+(n-2)(n-3)\left|\beta_{3}\right|\right)\left|a_{n}\right| \leq \lambda
$$

for some complex numbers $\beta_{1}, \beta_{2}, \beta_{3}$ and for some real $\lambda>0$, then $f(z) \in$ $\mathcal{D}\left(\beta_{1}, \beta_{2}, \beta_{3} ; \lambda\right)$.

Proof. We observe that

$$
\begin{aligned}
& \left|\beta_{1} z\left(\frac{D^{2} f(z)}{z}\right)^{\prime}+\beta_{2} z^{2}\left(\frac{D^{2} f(z)}{z}\right)^{\prime \prime}+\beta_{3} z^{3}\left(\frac{D^{2} f(z)}{z}\right)^{\prime \prime \prime}\right| \\
= & \left|\sum_{n=2}^{\infty} n^{2}(n-1)\left(\beta_{1}+(n-2) \beta_{2}+(n-2)(n-3) \beta_{3}\right) a_{n} z^{n-1}\right| \\
\leq & \sum_{n=2}^{\infty} n^{2}(n-1)\left(\left|\beta_{1}\right|+(n-2)\left|\beta_{2}\right|+(n-2)(n-3)\left|\beta_{3}\right|\right)\left|a_{n}\right||z|^{n-1} \\
< & \sum_{n=2}^{\infty} n^{2}(n-1)\left(\left|\beta_{1}\right|+(n-2)\left|\beta_{2}\right|+(n-2)(n-3)\left|\beta_{3}\right|\right)\left|a_{n}\right| .
\end{aligned}
$$

Therefore, if $f(z)$ satisfies the inequality (6), then $f(z) \in \mathcal{D}\left(\beta_{1}, \beta_{2}, \beta_{3} ; \lambda\right)$.

Next, we prove

Theorem 2.2. If $f(z) \in \mathcal{D}\left(\beta_{1}, \beta_{2}, \beta_{3} ; \lambda\right)$ with $\arg \beta_{1}=\arg \beta_{2}=\arg \beta_{3}=\phi$ and $a_{n}=\left|a_{n}\right| e^{i((n-1) \theta-\phi)}(n=2,3, \ldots)$, then we have

$$
\sum_{n=2}^{\infty} n^{2}(n-1)\left(\left|\beta_{1}\right|+(n-2)\left|\beta_{2}\right|+(n-2)(n-3)\left|\beta_{3}\right|\right)\left|a_{n}\right| \leq \lambda .
$$

Proof. For $f(z) \in \mathcal{D}\left(\beta_{1}, \beta_{2}, \beta_{3} ; \lambda\right)$, we see that

$$
\begin{aligned}
& \left|\beta_{1} z\left(\frac{D^{2} f(z)}{z}\right)^{\prime}+\beta_{2} z^{2}\left(\frac{D^{2} f(z)}{z}\right)^{\prime \prime}+\beta_{3} z^{3}\left(\frac{D^{2} f(z)}{z}\right)^{\prime \prime \prime}\right| \\
= & \left|\sum_{n=2}^{\infty} n^{2}(n-1)\left(\beta_{1}+(n-2) \beta_{2}+(n-2)(n-3) \beta_{3}\right) a_{n} z^{n-1}\right| \\
= & \left|\sum_{n=2}^{\infty} n^{2}(n-1)\left(\left|\beta_{1}\right|+(n-2)\left|\beta_{2}\right|+(n-2)(n-3)\left|\beta_{3}\right|\right)\right| a_{n}\left|e^{i(n-1) \theta} z^{n-1}\right| \\
\leq & \lambda .
\end{aligned}
$$


for all $z \in \mathcal{U}$. Let us consider a point $z \in \mathcal{U}$ such that $z=|z| e^{-i \theta}$. Then we have

$$
\sum_{n=2}^{\infty} n^{2}(n-1)\left(\left|\beta_{1}\right|+(n-2)\left|\beta_{2}\right|+(n-2)(n-3)\left|\beta_{3}\right|\right)\left|a_{n}\right||z|^{n-1} \leq \lambda .
$$

Letting $|z| \rightarrow 1^{-}$, we obtain

$$
\sum_{n=2}^{\infty} n^{2}(n-1)\left(\left|\beta_{1}\right|+(n-2)\left|\beta_{2}\right|+(n-2)(n-3)\left|\beta_{3}\right|\right)\left|a_{n}\right| \leq \lambda .
$$

Corollary 2.3. If $f(z) \in \mathcal{D}\left(\beta_{1}, \beta_{2}, \beta_{3} ; \lambda\right)$ with $\arg \beta_{1}=\arg \beta_{2}=\arg \beta_{3}=\phi$ and $a_{n}=\left|a_{n}\right| e^{i((n-1) \theta-\phi)}(n=2,3, \ldots)$, then we have

$$
\left|a_{n}\right| \leq \frac{\lambda}{n^{2}(n-1)\left(\left|\beta_{1}\right|+(n-2)\left|\beta_{2}\right|+(n-2)(n-3)\left|\beta_{3}\right|\right)} \quad(n=2,3, \ldots) .
$$

Example 2.4. Let us consider the function $f(z) \in \mathcal{D}\left(\beta_{1}, \beta_{2}, \beta_{3} ; \lambda\right)$ with $\arg \beta_{1}=$ $\arg \beta_{2}=\arg \beta_{3}=\phi$ and

$$
a_{n}=\frac{\lambda e^{i((n-1) \theta-\phi)}}{n^{3}(n-1)^{2}\left(\left|\beta_{1}\right|+(n-2)\left|\beta_{2}\right|+(n-2)(n-3)\left|\beta_{3}\right|\right)} \quad(n=2,3 \ldots) .
$$

Then we see that

$$
\begin{aligned}
& \sum_{n=2}^{\infty} n^{2}(n-1)\left(\left|\beta_{1}\right|+(n-2)\left|\beta_{2}\right|+(n-2)(n-3)\left|\beta_{3}\right|\right)\left|a_{n}\right| \\
= & \lambda \sum_{n=2}^{\infty} \frac{1}{n(n-1)}=\lambda \sum_{n=2}^{\infty}\left(\frac{1}{n-1}-\frac{1}{n}\right)=\lambda .
\end{aligned}
$$

Corollary 2.5. If $f(z) \in \mathcal{D}\left(\beta_{1}, \beta_{2}, \beta_{3} ; \lambda\right)$ with $\arg \beta_{1}=\arg \beta_{2}=\arg \beta_{3}=\phi$ and $a_{n}=\left|a_{n}\right| e^{i((n-1) \theta-\phi)}(n=2,3, \ldots)$, then we have

$$
|z|-\sum_{n=2}^{j}\left|a_{n}\right||z|^{n}-A_{j}|z|^{j+1} \leq|f(z)| \leq|z|+\sum_{n=2}^{j}\left|a_{n}\right||z|^{n}+A_{j}|z|^{j+1}
$$

with

$$
A_{j}=\frac{\left(\lambda-\sum_{n=2}^{j} n^{2}(n-1)\left(\left|\beta_{1}\right|+(n-2)\left|\beta_{2}\right|+(n-2)(n-3)\left|\beta_{3}\right|\right)\left|a_{n}\right|\right)}{j(j+1)^{2}\left(\left|\beta_{1}\right|+(j-1)\left|\beta_{2}\right|+(j-1)(j-2)\left|\beta_{3}\right|\right)}
$$


and

$$
1-\sum_{n=2}^{j}\left|a_{n}\right||z|^{n-1}-B_{j}|z|^{j} \leq\left|f^{\prime}(z)\right| \leq 1+\sum_{n=2}^{j}\left|a_{n}\right||z|^{n-1}+B_{j}|z|^{j}
$$

with

$$
B_{j}=\frac{\left(\lambda-\sum_{n=2}^{j} n^{2}(n-1)\left(\left|\beta_{1}\right|+(n-2)\left|\beta_{2}\right|+(n-2)(n-3)\left|\beta_{3}\right|\right)\left|a_{n}\right|\right)}{j(j+1)\left(\left|\beta_{1}\right|+(j-1)\left|\beta_{2}\right|+(j-1)(j-2)\left|\beta_{3}\right|\right)} .
$$

Proof. In view of Theorem 2.1, we know that

$$
\begin{aligned}
& \sum_{n=j+1}^{\infty} n^{2}(n-1)\left(\left|\beta_{1}\right|+(n-2)\left|\beta_{2}\right|+(n-2)(n-3)\left|\beta_{3}\right|\right)\left|a_{n}\right| \\
\leq & \lambda-\sum_{n=2}^{j} n^{2}(n-1)\left(\left|\beta_{1}\right|+(n-2)\left|\beta_{2}\right|+(n-2)(n-3)\left|\beta_{3}\right|\right)\left|a_{n}\right| .
\end{aligned}
$$

Further, we note that

$$
\begin{aligned}
& j(j+1)^{2}\left(\left|\beta_{1}\right|+(j-1)\left|\beta_{2}\right|+(j-1)(j-2)\left|\beta_{3}\right|\right) \sum_{n=j+1}^{\infty}\left|a_{n}\right| \\
\leq & \sum_{n=j+1}^{\infty} n^{2}(n-1)\left(\left|\beta_{1}\right|+(n-2)\left|\beta_{2}\right|+(n-2)(n-3)\left|\beta_{3}\right|\right)\left|a_{n}\right|,
\end{aligned}
$$

which is equivalent to

$$
\begin{aligned}
\sum_{n=j+1}^{\infty}\left|a_{n}\right| & \leq \frac{\left(\lambda-\sum_{n=2}^{j} n^{2}(n-1)\left(\left|\beta_{1}\right|+(n-2)\left|\beta_{2}\right|+(n-2)(n-3)\left|\beta_{3}\right|\right)\left|a_{n}\right|\right)}{j(j+1)^{2}\left(\left|\beta_{1}\right|+(j-1)\left|\beta_{2}\right|+(j-1)(j-2)\left|\beta_{3}\right|\right)} \\
& =A_{j} .
\end{aligned}
$$

Thus, we have

$$
|f(z)| \leq|z|+\sum_{n=2}^{j}\left|a_{n}\right||z|^{n}+\sum_{n=j+1}^{\infty}\left|a_{n}\right||z|^{n} \leq|z|+\sum_{n=2}^{j}\left|a_{n}\right||z|^{n}+A_{j}|z|^{j+1}
$$

and

$$
|f(z)| \geq|z|-\sum_{n=2}^{j}\left|a_{n}\right||z|^{n}-\sum_{n=j+1}^{\infty}\left|a_{n}\right||z|^{n} \geq|z|-\sum_{n=2}^{j}\left|a_{n}\right||z|^{n}-A_{j}|z|^{j+1} .
$$


Next, we observe that

$$
\begin{aligned}
& j(j+1)\left(\left|\beta_{1}\right|+(j-1)\left|\beta_{2}\right|+(j-1)(j-2)\left|\beta_{3}\right|\right) \sum_{n=j+1}^{\infty} n\left|a_{n}\right| \\
\leq & \sum_{n=j+1}^{\infty} n^{2}(n-1)\left(\left|\beta_{1}\right|+(n-2)\left|\beta_{2}\right|+(n-2)(n-3)\left|\beta_{3}\right|\right)\left|a_{n}\right| \\
\leq & \lambda-\sum_{n=2}^{j} n^{2}(n-1)\left(\left|\beta_{1}\right|+(n-2)\left|\beta_{2}\right|+(n-2)(n-3)\left|\beta_{3}\right|\right)\left|a_{n}\right|,
\end{aligned}
$$

which implies that

$$
\begin{aligned}
\sum_{n=j+1}^{\infty} n\left|a_{n}\right| & \leq \frac{\left(\lambda-\sum_{n=2}^{j} n^{2}(n-1)\left(\left|\beta_{1}\right|+(n-2)\left|\beta_{2}\right|+(n-2)(n-3)\left|\beta_{3}\right|\right)\left|a_{n}\right|\right)}{j(j+1)\left(\left|\beta_{1}\right|+(j-1)\left|\beta_{2}\right|+(j-1)(j-2)\left|\beta_{3}\right|\right)} \\
& =B_{j} .
\end{aligned}
$$

Therefore, we obtain that

$$
\left|f^{\prime}(z)\right| \leq 1+\sum_{n=2}^{j} n\left|a_{n}\right||z|^{n-1}+\sum_{n=j+1}^{\infty} n\left|a_{n}\right||z|^{n-1} \leq 1+\sum_{n=2}^{j}\left|a_{n}\right||z|^{n-1}+B_{j}|z|^{j}
$$

and

$$
\left|f^{\prime}(z)\right| \geq 1-\sum_{n=2}^{j} n\left|a_{n}\right||z|^{n-1}-\sum_{n=j+1}^{\infty} n\left|a_{n}\right||z|^{n-1} \geq 1-\sum_{n=2}^{j}\left|a_{n}\right||z|^{n-1}-B_{j}|z|^{j}
$$

\section{Radius Problem for the Class $\mathcal{G}(\theta, \alpha)$}

To obtain the radius problem for the class $\mathcal{G}(\theta, \alpha)$, we need the following lemma.

Lemma 3.1. If $f(z) \in \mathcal{G}(\theta, \alpha)$, then

$$
\sum_{n=2}^{\infty} n^{3}\left|a_{n}\right| \leq 1-\alpha
$$

Proof. Let $f(z) \in \mathcal{G}(\theta, \alpha)$. Then, we have

$$
\begin{aligned}
\operatorname{Re}\left\{\frac{D^{3} \mathrm{f}(\mathrm{z})}{\mathrm{z}}\right\} & =\operatorname{Re}\left\{1+\sum_{\mathrm{n}=2}^{\infty} \mathrm{n}^{3} \mathrm{a}_{\mathrm{n}} \mathrm{z}^{\mathrm{n}-1}\right\}=\operatorname{Re}\left\{1+\sum_{\mathrm{n}=2}^{\infty} \mathrm{n}^{3}\left|\mathrm{a}_{\mathrm{n}}\right| \mathrm{e}^{\mathrm{i}((\mathrm{n}-1) \theta+\pi)} \mathrm{z}^{\mathrm{n}-1}\right\} \\
& =\operatorname{Re}\left\{1-\sum_{\mathrm{n}=2}^{\infty} \mathrm{n}^{3}\left|\mathrm{a}_{\mathrm{n}}\right| \mathrm{e}^{\mathrm{i}((\mathrm{n}-1) \theta} \mathrm{z}^{\mathrm{n}-1}\right\}>\alpha
\end{aligned}
$$


for all $z \in \mathcal{U}$. Let us consider a point $z \in \mathcal{U}$ such that $z=|z| e^{-i \theta}$. Then we have

$$
1-\sum_{n=2}^{\infty} n^{3}\left|a_{n}\right||z|^{n-1}>\alpha
$$

Letting $|z| \rightarrow 1^{-}$, we obtain the inequality (7).

Corollary 3.2. If $f(z) \in \mathcal{G}(\theta, \alpha)$, then

$$
\left|a_{n}\right| \leq \frac{1-\alpha}{n^{3}} \quad(n=2,3, \ldots) .
$$

Remark 3.3. By Lemma 3.1, we observe that if $f(z) \in \mathcal{G}(\theta, \alpha)$, then

$$
\sum_{n=2}^{\infty} n^{2}(n-1)\left|a_{n}\right| \leq \sum_{n=2}^{\infty} n^{3}\left|a_{n}\right| \leq 1-\alpha .
$$

Applying Theorem 2.1 and Lemma 3.1, we derive

Theorem 3.4. If $f(z) \in \mathcal{G}(\theta, \alpha)$, and $\delta \in \mathbb{C}(0<|\delta|<1)$. Then the function $\frac{1}{\delta} f(\delta z) \in \mathcal{D}\left(\beta_{1}, \beta_{2}, \beta_{3} ; \lambda\right)$ for $\left(0<|\delta| \leq\left|\delta_{0}(\lambda)\right|\right.$, where $\left|\delta_{0}(\lambda)\right|$ is the smallest positive root of the equation

$$
\begin{aligned}
& \left|\beta_{1}\right| \frac{|\delta| \sqrt{2\left(|\delta|^{2}+2\right)(1-\alpha)}}{\left(1-|\delta|^{2}\right)^{2}} \\
& \quad+\left|\beta_{2}\right| \frac{|\delta|^{2} \sqrt{6\left(3|\delta|^{4}+14|\delta|^{2}+3\right)\left(1-\alpha-4\left|a_{2}\right|^{2}\right)}}{\left(1-|\delta|^{2}\right)^{3}} \\
& +\left|\beta_{3}\right| \frac{|\delta|^{3} \sqrt{48\left(6|\delta|^{6}+52|\delta|^{4}+43|\delta|^{2}+4\right)\left(1-\alpha-4\left|a_{2}\right|^{2}-18\left|a_{3}\right|^{2}\right)}}{\left(1-|\delta|^{2}\right)^{4}} \\
= & \lambda
\end{aligned}
$$

in $0<|\delta|<1$.

Proof. For $f(z) \in \mathcal{G}(\theta, \alpha)$, we see that

$$
\frac{1}{\delta} f(\delta z)=z+\sum_{n=2}^{\infty} \delta^{n-1} a_{n} z^{n}
$$

and

$$
\sum_{n=2}^{\infty} n^{2}(n-1)\left|a_{n}\right|^{2} \leq 1-\alpha .
$$


Thus, to show that $\frac{1}{\delta} f(\delta z) \in \mathcal{D}\left(\beta_{1}, \beta_{2}, \beta_{3} ; \lambda\right)$, from Theorem 2.1, it is sufficient to prove that

$$
\sum_{n=2}^{\infty} n^{2}(n-1)\left(\left|\beta_{1}\right|+(n-2)\left|\beta_{2}\right|+(n-2)(n-3)\left|\beta_{3}\right|\right)|\delta|^{n-1}\left|a_{n}\right| \leq \lambda .
$$

Applying Cauchy-Schwarz inequality, we note that

$$
\begin{aligned}
& \sum_{n=2}^{\infty} n^{2}(n-1)\left(\left|\beta_{1}\right|+(n-2)\left|\beta_{2}\right|+(n-2)(n-3)\left|\beta_{3}\right|\right)|\delta|^{n-1}\left|a_{n}\right| \\
\leq & \frac{\left|\beta_{1}\right|}{|\delta|}\left(\sum_{n=2}^{\infty} n^{2}(n-1)|\delta|^{2 n}\right)^{\frac{1}{2}}\left(\sum_{n=2}^{\infty} n^{2}(n-1)\left|a_{n}\right|^{2}\right)^{\frac{1}{2}} \\
& +\frac{\left|\beta_{2}\right|}{|\delta|}\left(\sum_{n=3}^{\infty} n^{2}(n-1)(n-2)^{2}|\delta|^{2 n}\right)^{\frac{1}{2}}\left(\sum_{n=3}^{\infty} n^{2}(n-1)\left|a_{n}\right|^{2}\right)^{\frac{1}{2}} \\
& +\frac{\left|\beta_{3}\right|}{|\delta|}\left(\sum_{n=4}^{\infty} n^{2}(n-1)(n-2)^{2}(n-3)^{2}|\delta|^{2 n}\right)^{\frac{1}{2}}\left(\sum_{n=4}^{\infty} n^{2}(n-1)\left|a_{n}\right|^{2}\right)^{\frac{1}{2}} \\
\leq & \frac{\left|\beta_{1}\right|}{|\delta|}\left(\sum_{n=2}^{\infty} n^{2}(n-1)|\delta|^{2 n}\right)^{\frac{1}{2}} \sqrt{1-\alpha} \\
& +\frac{\left|\beta_{2}\right|}{|\delta|}\left(\sum_{n=3}^{\infty} n^{2}(n-1)(n-2)^{2}|\delta|^{2 n}\right)^{\frac{1}{2}} \sqrt{1-\alpha-4\left|a_{2}\right|^{2}} \\
& +\frac{\left|\beta_{3}\right|}{|\delta|}\left(\sum_{n=4}^{\infty} n^{2}(n-1)(n-2)^{2}(n-3)^{2}|\delta|^{2 n}\right)^{\frac{1}{2}} \sqrt{1-\alpha-4\left|a_{2}\right|^{2}-18\left|a_{3}\right|^{2}} .
\end{aligned}
$$

Making use of

$$
\sum_{n=0}^{\infty} x^{n}=\frac{1}{1-x}, \quad(|x|<1)
$$

we have

$$
\sum_{n=2}^{\infty} n^{2}(n-1) x^{n}=\frac{2 x^{2}(x+2)}{(1-x)^{4}}
$$

Since

$$
\sum_{n=3}^{\infty}(n-2) x^{n-1}=x^{2}\left(\sum_{n=3}^{\infty}(n-2) x^{n-3}\right)=x^{2}\left(\sum_{n=3}^{\infty} x^{n-2}\right)^{\prime}=\frac{x^{2}}{(1-x)^{2}},
$$


we see that

$$
\sum_{n=3}^{\infty}(n-1)(n-2)^{2} x^{n}=x^{3}\left(\frac{x^{2}}{(1-x)^{2}}\right)^{\prime \prime}=\frac{2 x^{3}+4 x^{4}}{(1-x)^{4}} .
$$

and thus, we obtain

$$
\sum_{n=3}^{\infty} n(n-1)(n-2)^{2} x^{n}=\frac{6 x^{3}+18 x^{4}}{(1-x)^{5}}
$$

which yields

$$
\sum_{n=3}^{\infty} n^{2}(n-1)(n-2)^{2} x^{n}=\frac{6 x^{3}\left(3 x^{2}+14 x+3\right)}{(1-x)^{6}} .
$$

Furthermore, we have

$$
\begin{aligned}
\sum_{n=4}^{\infty}(n-1)(n-2)^{2}(n-3)^{2} x^{n} & =x^{4}\left(\sum_{n=4}^{\infty}(n-1)(n-2)^{2}(n-3)^{2} x^{n-4}\right) \\
& =x^{4}\left(\sum_{n=4}^{\infty}(n-2)(n-3) x^{n-1}\right)^{\prime \prime \prime},
\end{aligned}
$$

but

$$
\sum_{n=4}^{\infty}(n-2)(n-3) x^{n-1}=x^{3}\left(\sum_{n=4}^{\infty}(n-2)(n-3) x^{n-4}\right)=\frac{2 x^{3}}{(1-x)^{3}}
$$

thus, we have

$$
\sum_{n=4}^{\infty}(n-1)(n-2)^{2}(n-3)^{2} x^{n}=\frac{12 x^{4}+72 x^{5}+36 x^{6}}{(1-x)^{6}},
$$

which yields

$$
\sum_{n=4}^{\infty} n(n-1)(n-2)^{2}(n-3)^{2} x^{n}=\frac{48 x^{4}+384 x^{5}+288 x^{6}}{(1-x)^{7}} .
$$

This gives us that

$$
\sum_{n=4}^{\infty} n^{2}(n-1)(n-2)^{2}(n-3)^{2} x^{n}=\frac{48 x^{4}\left(6 x^{3}+52 x^{2}+43 x+4\right)}{(1-x)^{8}} .
$$


Therefore, from (9)- (12) with $|\delta|^{2}=x$, we obtain

$$
\begin{aligned}
& \sum_{n=2}^{\infty} n^{2}(n-1)\left(\left|\beta_{1}\right|+(n-2)\left|\beta_{2}\right|+(n-2)(n-3)\left|\beta_{3}\right|\right)|\delta|^{n-1}\left|a_{n}\right| \\
\leq & \left|\beta_{1}\right| \frac{|\delta| \sqrt{2\left(|\delta|^{2}+2\right)(1-\alpha)}}{\left(1-|\delta|^{2}\right)^{2}} \\
& +\left|\beta_{2}\right| \frac{|\delta|^{2} \sqrt{6\left(3|\delta|^{4}+14|\delta|^{2}+3\right)\left(1-\alpha-4\left|a_{2}\right|^{2}\right)}}{\left(1-|\delta|^{2}\right)^{3}} \\
& +\left|\beta_{3}\right| \frac{|\delta|^{3} \sqrt{48\left(6|\delta|^{6}+52|\delta|^{4}+43|\delta|^{2}+4\right)\left(1-\alpha-4\left|a_{2}\right|^{2}-18\left|a_{3}\right|^{2}\right)}}{\left(1-|\delta|^{2}\right)^{4}}
\end{aligned}
$$

Now, let us consider the complex number $\delta(0<|\delta|<1)$ such that

$$
\begin{array}{ll} 
& \left|\beta_{1}\right| \frac{|\delta| \sqrt{2\left(|\delta|^{2}+2\right)(1-\alpha)}}{\left(1-|\delta|^{2}\right)^{2}} \\
+ & \left|\beta_{2}\right| \frac{|\delta|^{2} \sqrt{6\left(3|\delta|^{4}+14|\delta|^{2}+3\right)\left(1-\alpha-4\left|a_{2}\right|^{2}\right)}}{\left(1-|\delta|^{2}\right)^{3}} \\
+ & +\beta_{3} \mid \frac{|\delta|^{3} \sqrt{48\left(6|\delta|^{6}+52|\delta|^{4}+43|\delta|^{2}+4\right)\left(1-\alpha-4\left|a_{2}\right|^{2}-18\left|a_{3}\right|^{2}\right)}}{\left(1-|\delta|^{2}\right)^{4}} \\
= & \lambda
\end{array}
$$

If we define the function $h(|\delta|)$ by

$$
\begin{aligned}
h(|\delta|)= & \left|\beta_{1}\right||\delta|\left(1-|\delta|^{2}\right)^{2} \sqrt{2\left(|\delta|^{2}+2\right)(1-\alpha)} \\
& +\left|\beta_{2}\right||\delta|^{2}\left(1-|\delta|^{2}\right) \sqrt{6\left(3|\delta|^{4}+14|\delta|^{2}+3\right)\left(1-\alpha-4\left|a_{2}\right|^{2}\right)} \\
& +\left|\beta_{3}\right||\delta|^{3} \sqrt{48\left(6|\delta|^{6}+52|\delta|^{4}+43|\delta|^{2}+4\right)\left(1-\alpha-4\left|a_{2}\right|^{2}-18\left|a_{3}\right|^{2}\right)} \\
& -\lambda\left(1-|\delta|^{2}\right)^{4}
\end{aligned}
$$

then we have $h(0)=-\lambda<0$ and $h(1)=4 \sqrt{3}\left|\beta_{3}\right| \sqrt{105\left(1-\alpha-4\left|a_{2}\right|^{2}-18\left|a_{3}\right|^{2}\right)}>$ 0 . This means that there exists some $\delta_{0}$ such that $h\left(\left|\delta_{0}\right|\right)=0\left(0<\left|\delta_{0}\right|<1\right)$. This completes the proof of the theorem. 


\section{Radius Problem for the Class $Q(\theta, \alpha)$}

For the class $Q(\theta, \alpha)$, we prove the following lemma.

Lemma 4.1. If $f(z) \in \mathcal{Q}(\theta, \alpha)$, then

$$
\sum_{n=2}^{\infty} n(n-\alpha)\left|a_{n}\right| \leq 1-\alpha .
$$

Proof. Let $f(z) \in \mathcal{Q}(\theta, \alpha)$. Then, we have

$$
\begin{aligned}
\operatorname{Re}\left\{\frac{D^{2} f(z)}{D^{1} f(z)}\right\} & =\operatorname{Re}\left\{\frac{1+\sum_{n=2}^{\infty} n^{2} a_{n} z^{n-1}}{1+\sum_{n=2}^{\infty} n a_{n} z^{n-1}}\right\} \\
& =\operatorname{Re}\left\{\frac{1-\sum_{n=2}^{\infty} n^{2}\left|a_{n}\right| e^{i(n-1) \theta} z^{n-1}}{1-\sum_{n=2}^{\infty} n\left|a_{n}\right| e^{i(n-1) \theta} z^{n-1}}\right\}>\alpha
\end{aligned}
$$

for all $z \in \mathcal{U}$. Let us consider a point $z \in \mathcal{U}$ such that $z=|z| e^{-i \theta}$. Then we have

$$
\frac{1-\sum_{n=2}^{\infty} n^{2}\left|a_{n}\right||z|^{n-1}}{1-\sum_{n=2}^{\infty} n\left|a_{n}\right||z|^{n-1}}>\alpha
$$

Letting $|z| \rightarrow 1^{-}$, we obtain the inequality (13).

Corollary 4.2. If $f(z) \in \mathcal{Q}(\theta, \alpha)$, then

$$
\left|a_{n}\right| \leq \frac{1-\alpha}{n(n-\alpha)} \quad(n=2,3, \ldots) .
$$

Remark 4.3. If $f(z) \in \mathcal{Q}(\theta, \alpha)$, then

$$
\sum_{n=2}^{\infty} n^{2}(n-1)\left|a_{n}\right| \leq \sum_{n=2}^{\infty} n(n-\alpha)\left|a_{n}\right| \leq 1-\alpha .
$$

Applying Theorem 2.1, Lemma 4.1 and using the same technique as in the proof of Theorem 3.4, we derive

Theorem 4.4. If $f(z) \in \mathcal{Q}(\theta, \alpha)$, and $\delta \in \mathbb{C}(0<|\delta|<1)$. Then the function $\frac{1}{\delta} f(\delta z) \in \mathcal{D}\left(\beta_{1}, \beta_{2}, \beta_{3} ; \lambda\right)$ for $\left(0<|\delta| \leq\left|\delta_{0}(\lambda)\right|\right.$, where $\left|\delta_{0}(\lambda)\right|$ is the smallest positive 
root of the equation

$$
\begin{aligned}
& \left|\beta_{1}\right| \frac{|\delta| \sqrt{2\left(|\delta|^{2}+2\right)(1-\alpha)}}{\left(1-|\delta|^{2}\right)^{2}} \\
(14) \quad & +\left|\beta_{2}\right| \frac{|\delta|^{2} \sqrt{6\left(3|\delta|^{4}+14|\delta|^{2}+3\right)\left(1-\alpha-4\left|a_{2}\right|^{2}\right)}}{\left(1-|\delta|^{2}\right)^{3}} \\
& +\left|\beta_{3}\right| \frac{|\delta|^{3} \sqrt{48\left(6|\delta|^{6}+52|\delta|^{4}+43|\delta|^{2}+4\right)\left(1-\alpha-4\left|a_{2}\right|^{2}-18\left|a_{3}\right|^{2}\right)}}{\left(1-|\delta|^{2}\right)^{4}} \\
= & \lambda
\end{aligned}
$$

in $0<|\delta|<1$.

\section{References}

[1] G. S. Sãlãgean, Subclasses of univalent functions, Lecture Notes in Math. SpringerVerlag, 1013(1983), 362-372.

[2] Neslihan Uyanik and Shigeyoshi Owa, New extensions for classes of analytic functions associated with close-to-convex and starlike of order $\alpha$, Mathematical and Computer Modelling, 54(2011), 359-366. 